\title{
SOS Methods for Stability Analysis of Neutral Differential Systems
}

\author{
Matthew M. Peet ${ }^{1}$, Catherine Bonnet ${ }^{1}$, and Hitay Özbay ${ }^{2}$ \\ 1 M. M. Peet and C. Bonnet are with INRIA-Rocquencourt, Domaine de Voluceau, \\ Rocquencourt BP105 78153, Le Chesnay Cedex, France. matthew. peet@inria.fr, \\ catherine.bonneteinria.fr. \\ 2 H. Özbay is with the Department of Electrical and Electronics Engineering, Bilkent \\ University, Bilkent, 06800 Ankara, Turkey hitay@bilkent. edu.tr.
}

\begin{abstract}
Summary. This paper gives a description of how "sum-of-squares" (SOS) techniques can be used to check frequency-domain conditions for the stability of neutral differential systems. For delay-dependent stability, we adapt an approach of Zhang et al. [10] and show how the associated conditions can be expressed as the infeasibility of certain semialgebraic sets. For delay-independent stability, we propose an alternative method of reducing the problem to infeasibility of certain semialgebraic sets. Then, using Positivstellensatz results from semi-algebraic geometry, we convert these infeasibility conditions to feasibility problems using sum-of-squares variables. By bounding the degree of the variables and using the Matlab toolbox SOSTOOLS [7], these conditions can be checked using semidefinite programming
\end{abstract}

\section{Introduction}

In this paper, we consider the problem of verification of certain frequency-domain tests for stability of delay systems of the neutral type. We show that several frequency-domain conditions can be reduced to optimization problems on the cone of positive semidefinite matrices. Such an approach is an alternative to the classical graphical tests (e.g. the Nyquist criterion). The motivation for using optimization-based methods is as follows

a) The computational complexity of these methods is well-established. Computational complexity provides a standard benchmark for the difficulty of a given problem. A condition which is expressed as an optimization problem, therefore, will have well-known computational properties.

b) While the accuracy of a result based on graphical methods may be limited by the resolution and range of the plot, a feasible result from an optimization-based method serves as a readily verifiable certificate of stability.

The use of frequency domain criteria for analysis of linear systems has an extensive history and we will not make an attempt to catalogue the list of accomplishments in this field. We do note, however, that while for finite-dimensional systems, time-domain-based LMI methods currently compete successfully with frequency-domain-based graphical criteria, the same can not be said to be true for infinite-dimensional systems.

J.J. Loiseau et al. (Eds.): Topics in Time Delay Systems, LNCIS 388, pp. $97-107$. springerlink.com

(c) Springer-Verlag Berlin Heidelberg 2009 
In [2], we have already considered some simple delay-independent stability conditions for systems of the neutral type using SOS techniques. Our aim here is to develop more sophisticated methods which will allow us to check the delay-dependant $H_{\infty}$-stability of neutral systems. The paper is organized as follows. We begin in section 2 by recalling some background on polynomial optimization and "sum-of-squares".

In section 3, we show how a method based on dichotomy arguments which was proposed by Zhang et al [10] for the stability analysis of retarded-type delay systems can also be applied to the case of neutral-type systems. The main theorems which enable us to formulate delay-dependant stability in terms of feasibility of semi-algebraic sets and SOS polynomials conditions are given. It is shown that the problem of the appearance of asymptotic chains of roots, common for neutral systems, is solved by the Zhang method of rational overapproximation. In Section 4 we give some numerical examples which show the efficacy of the proposed method. Finally a conclusion is given in section 5

\section{The Positivstellensatz and Sum-of-Squares}

A polynomial, $p$, is said to be positive on $G \subset \mathbb{R}^{n}$ if

$$
p(x) \geq 0 \text { for all } x \in G .
$$

If $G$ is not mentioned, then it is assumed $G=\mathbb{R}^{n}$. A semialgebraic set is a subset of $\mathbb{R}^{n}$ defined by polynomials $p_{i}$, as

$$
G:=\left\{x \in \mathbb{R}^{n}: p_{i}(x) \geq 0, i=1, \ldots, k\right\} .
$$

Given a polynomial, the question of whether it is positive has been shown to be NP-hard. A polynomial, $p$, is said to be sum-of-squares (SOS) in variables $x$, denoted $p \in \Sigma_{s}[x]$ if there exist a finite number of other polynomials, $g_{i}$ such that

$$
p(x)=\sum_{i=1}^{k} g_{i}(x)^{2} .
$$

A sum-of-squares polynomial is positive, but a positive polynomial may not be sum-ofsquares. A necessary and sufficient condition for the existence of a sum-of-squares representation for a polynomial, $p$, of degree $2 d$ is the existence of a positive semidefinite matrix, $Q$, such that

$$
p(x)=Z(x)^{T} Q Z(x),
$$

where $Z$ is any vector whose elements form a basis for the polynomials of degree $d$. Positivstellensatz results are "theorems of the alternative" which say that either a semialgebraic set is feasible or there exists a sum-of-squares refutation of feasibility. The Positivstellensatz that we use in this paper is that given by Stengle [9].

Theorem 1 (Stengle). The following are equivalent

1.

$$
\left\{x: \begin{array}{l}
p_{i}(x) \geq 0 \quad i=1, \ldots, k \\
q_{j}(x)=0 j=1, \ldots, m
\end{array}\right\}=\emptyset
$$


2. There exist $t_{i} \in \mathbb{R}[x], s_{i}, r_{i j}, \ldots \in \Sigma_{s}[x]$ such that

$$
-1=\sum_{i} q_{i} t_{i}+s_{0}+\sum_{i} s_{i} p_{i}+\sum_{i \neq j} r_{i j} p_{i} p_{j}+\cdots
$$

We use $\mathbb{R}[x]$ to denote the real-valued polynomials in variables $x$. For a given degree bound, the conditions associated with Stengle's Positivstellensatz can be represented as a semidefinite program. Note that, in general, no such upper bound on the degree bound will be known a-priori.

\section{Stability of Neutral-Type Systems}

In this section, we consider aspects of the stability of general neutral-type systems of the form

$$
\dot{x}(t)=\sum_{i=1}^{m} B_{i} \dot{x}\left(t-\tau_{i}\right)+\sum_{j=0}^{m} A_{j} x\left(t-\tau_{j}\right)
$$

where $A_{i}, B_{j} \in \mathbb{R}^{n \times n}, \tau_{0}=0$ and $\tau_{i} \geq 0$.

\subsection{Dichotomy Methods}

The term dichotomy is used to describe methods based on the bisection of the complex plane into right and left half-planes. These methods are based on a continuity argument that states that if a system is stable for one value of a parameter and unstable for another value, then for some intermediate value of the parameter, the system must have a pole on the imaginary axis. For retarded and for neutral-type systems which satisfy certain conditions, this argument is valid due to the following theorem from Datko [3]. Let

$$
G_{\alpha}(s):=\left[s\left(I-\sum_{i=1}^{m} B_{i} e^{-\alpha s \gamma_{i}}\right)-\sum_{j=0}^{m} A_{j} e^{-\alpha s \gamma_{j}}\right] .
$$

Theorem 2 (Datko). Consider $G_{\alpha}$ as given by Equation [2]. If

$$
\operatorname{det}\left[I-\sum_{i=1}^{m} B_{i} e^{-s \gamma_{i}}\right]=0
$$

has all roots lying in some left half-plane $\operatorname{Re} s \in\left(-\infty,-\beta_{0}\right], \beta_{0}>0$, then

$$
\sigma\left(G_{\alpha}\right):=\sup _{\operatorname{det} G_{\alpha}(s)=0} \operatorname{Re} s
$$

is continuous on $\alpha \in[0, \infty)$.

In general, a system of the form 1 will define a transfer function. The poles of this transfer function will be defined by the roots of $\operatorname{det} G(s):=\sum_{i=0}^{n} q_{i}(s) e^{-\tau_{i} s}$ where $G_{\alpha}$ is given by Equation (2). Here the $q_{i}$ are complex polynomials with $\operatorname{deg} q_{0} \geq \operatorname{deg} q_{i}$ for $i=1, \ldots, n$. In general, for neutral-type systems, $\operatorname{deg} q_{0}=\operatorname{deg} q_{i}$ for at least one $i \geq 1$. 


\subsection{Generalization of a method of Zhang et al.}

In this subsection, we consider the approach of [10], wherein the exponential term is "covered" by a set of rational transfer functions parameterized by a single parameter. The size of the set, or the range of values of the parameter, is determined by the degree of the rational functions. We begin with the definition of the Padé approximate of $e^{-s}$.

$$
R_{m}(s)=\frac{P_{m}(s)}{P_{m}(-s)}
$$

where

$$
P_{m}(s)=\sum_{k=0}^{m} \frac{(2 m-k) ! m !(-s)^{k}}{(2 m) ! k !(m-k) !} .
$$

Now define the sets of irrational and rational functions.

$$
\begin{array}{r}
\Omega_{d}(\omega, h):=\left\{e^{-\imath \tau \omega}: \tau \in[0, h]\right\} \\
\Omega_{m}(\omega, h):=\left\{R_{m}\left(\imath \alpha_{m} \tau \omega\right): \tau \in[0, h]\right\}
\end{array}
$$

Where $\alpha_{m}:=\frac{1}{2 \pi} \min \left\{\omega>0 \mid R_{m}(\imath \omega)=1\right\}$. For example, $\alpha_{3}=1.2329, \alpha_{4}=1.0315$, and $\alpha_{5}=1.00363$.

The following is a key result of [10].

Lemma 13 (Zhang et al.). For every integer $m \geq 3$, the following statements hold.

1. All poles of $R_{m}(s)$ are in the open left half complex plane.

2. $\Omega_{d}(\omega, h) \subset \Omega_{m}(\omega, h)$ for any $h \geq 0$ and $\omega \geq 0$.

3. $\lim _{m \rightarrow \infty} \alpha_{m}=0$

Typically, Lemma 13 is used to prove that a delay-differential system has no poles in the closed right half-plane for an interval of delay of the form $[0, h]$. This is illustrated by the following theorem.

Theorem 3. Suppose $G$, as given by Equation (2), satisfies the conditions of Theorem 2 and $\operatorname{det} G(s):=\sum_{i=0}^{n} q_{i}(s) e^{-\tau_{i} s}$. Let $m \geq 3$, and suppose that

$$
\left\{s \in \mathcal{C}: \sum_{i=0}^{n} q_{i}(s)=0, \operatorname{Re} s \geq 0\right\}=\emptyset
$$

and

$$
\left\{\omega \geq 0, \tau_{i} \in\left[0, h_{i}\right]: \sum_{i=0}^{n} q_{i}(\omega \imath) R_{m}\left(\alpha_{m} \tau_{i} \omega \imath\right)=0\right\}=\emptyset .
$$

Then

$$
\left\{s \in \mathcal{C}, \tau_{i} \in\left[0, h_{i}\right]: \sum_{i=0}^{n} q_{i}(s) e^{-\tau_{i} s}=0, \operatorname{Re} s \geq 0\right\}=\emptyset .
$$

Theorem 3 is a trivial generalization of the work of Zhang et al. [10] to neutral-type systems. For retarded-type systems, the work of [10] proposed the construction of a parameterdependent state-space system. In a later work, [1] proposed the use of a generalized version of the KYP lemma to check the conditions associated with the retarded case through the construction of a perturbed singular system. In this paper, we use a "sum-of-squares" approach based on the application of the Positivstellensatz results described in Section 2 
For convenience, define the following functions.

$$
\begin{aligned}
& g_{r}(\omega, \tau):=\operatorname{Re}\left(\sum_{i=0}^{n} q_{i}(\omega \imath) P_{m}\left(\alpha_{m} \tau_{i} \omega \imath\right) \prod_{\substack{j=0 \\
j \neq i}}^{n} P_{m}\left(-\alpha_{m} \tau_{j} \omega \imath\right)\right) \\
& g_{i}(\omega, \tau):=\operatorname{Im}\left(\sum_{i=0}^{n} q_{i}(\omega \imath) P_{m}\left(\alpha_{m} \tau_{i} \omega \imath\right) \prod_{\substack{j=0 \\
j \neq i}}^{n} P_{m}\left(-\alpha_{m} \tau_{j} \omega \imath\right)\right)
\end{aligned}
$$

Lemma 14. The following are equivalent

1. The following set is infeasible.

$$
\left\{\omega \geq 0, \tau_{i} \in\left[0, h_{i}\right]: \sum_{i=0}^{n} q_{i}(\omega \imath) R_{m}\left(\alpha_{m} \tau_{i} \omega \imath\right)=0\right\}
$$

2. The following real semi-algebraic set is infeasible.

$$
\left\{\omega, \tau_{i} \in \mathbb{R}: \omega \geq 0, \tau\left(h_{i}-\tau_{i}\right) \geq 0, g_{r}\left(\omega, \tau_{i}\right)=0, g_{i}\left(\omega, \tau_{i}\right)=0\right\}
$$

3. There exist polynomials $t_{1}, t_{2} \in \mathbb{R}[\omega, \tau]$ and SOS polynomials $s_{i} \in \Sigma_{s}[\omega, \tau]$ such that

$$
\begin{aligned}
-1= & s_{0}+t_{1} g_{r}+t_{2} g_{i}+\omega s_{1}+\tau_{0}\left(h_{0}-\tau_{0}\right) s_{2} \\
& +\omega \tau_{0}\left(h_{0}-\tau_{0}\right) s_{3}+\omega \tau_{1}\left(h_{1}-\tau_{1}\right) s_{4}+\cdots
\end{aligned}
$$

Proof. By Lemma 13, all roots of $P_{m}(-s)$ are in the open left half complex plane, and so Statement 1 is equivalent to infeasibility of the following set.

$$
\left\{\omega \geq 0, \tau_{i} \in\left[0, h_{i}\right]: \sum_{i=0}^{n} q_{i}(\omega \imath) P_{m}\left(\alpha_{m} \tau_{i} \omega \imath\right) \prod_{\substack{j=0 \\ j \neq i}}^{n} P_{m}\left(-\alpha_{m} \tau_{j} \omega \imath\right)=0\right\}
$$

Now, $\tau \in[0, h]$ is equivalent to $\tau(h-\tau) \geq 0$ and so Statement 1 is equivalent to infeasibility of the set in Statement 2 Furthermore, the real or imaginary part of a complex polynomial is a polynomial in the real and complex parts of the complex argument. Therefore, the set in Statement2 is real semi-algebraic.

That Statement 2 is equivalent to Statement 3 is an immediate consequence of Theorem 1

We now give delay-dependent stability conditions for neutral-type systems.

Lemma 15. Define

$$
r_{m}:=\inf \left\{\left\|\sum_{i=0}^{n} q_{i}(\omega \imath) R_{m}\left(\alpha_{m} \tau_{i} \omega \imath\right)\right\|: \omega \geq 0, \tau_{i} \in\left[0, h_{i}\right]\right\}
$$

and

$$
r_{e}:=\inf \left\{\left\|\sum_{i=0}^{n} q_{i}(\omega \imath) e^{-\tau_{i} \omega \imath}\right\|: \omega \geq 0, \tau_{i} \in\left[0, h_{i}\right]\right\} .
$$

Then for any $m \geq 3, r_{e} \geq r_{m}$. 
Proof. Suppose there exists an $\omega_{w} \geq 0$ and $\tau_{i, w} \in\left[0, h_{i}\right]$ such that

$$
w=\left\|\sum_{i=0}^{n} q_{i}\left(\omega_{w} \imath\right) e^{-\tau_{i, w} \omega_{w} \imath}\right\|
$$

Now, by Lemma 13 there exists $\tau_{i, w}^{\prime} \in\left[0, h_{i}\right]$ such that

$$
\left\|\sum_{i=0}^{n} q_{i}\left(\omega_{w} \imath\right) R_{m}\left(\alpha_{m} \tau_{i, w}^{\prime} \omega_{w} \imath\right)\right\|=\left\|\sum_{i=0}^{n} q_{i}\left(\omega_{w} \imath\right) e^{-\tau_{i, w} \omega_{w} \imath}\right\|=w
$$

Therefore, $r_{m} \leq w$ and hence $r_{m} \leq r_{e}$.

Theorem 4. Suppose $G_{\tau}$, given by Equation (2), satisfies the conditions of Theorem 2 and $\operatorname{det} G_{\tau}(s):=\sum_{i=0}^{n} q_{i}(s) e^{-\tau_{i} s}$. Let $m \geq 3$ and suppose that

$$
\left\{s \in \mathcal{C}: \sum_{i=0}^{n} q_{i}(s)=0, \operatorname{Re} s \geq 0\right\}=\emptyset
$$

and that there exist polynomials $t_{1}, t_{2} \in \mathbb{R}[\omega, \tau]$ and SOS polynomials $s_{i} \in \Sigma_{s}[\omega, \tau]$ such that

$$
\begin{aligned}
-1= & s_{0}+t_{1} g_{r}+t_{2} g_{i}+\omega s_{1}+\tau_{1}\left(h_{1}-\tau_{1}\right) s_{2} \\
& +\omega \tau_{1}\left(h_{1}-\tau_{1}\right) s_{3}+\omega \tau_{2}\left(h_{2}-\tau_{2}\right) s_{4}+\cdots
\end{aligned}
$$

Then the system defined by Equation 1 is $H_{\infty}$ stable for all $\tau_{i} \in\left[0, h_{i}\right]$

Proof. If the conditions of the theorem are satisfied, then by Lemma 14

$$
H_{m}(s):=\sum_{i=0}^{n} q_{i}(s) R_{m}\left(\alpha_{m} \tau_{i} s\right)
$$

has no roots on the imaginary axis for any $\tau_{i} \in\left[0, h_{i}\right]$. Therefore, by Theorem $3 \operatorname{det} G_{\tau}(s)$ has no roots on the closed right half plane. Therefore $\operatorname{det} G_{\tau}(s)^{-1}$ is analytic on the closed right half plane. It remains to show that $\operatorname{det} G_{\tau}(s)^{-1}$ is bounded on the imaginary axis.

Since $H_{m}$ is a polynomial, then for any $\tau_{i} \in\left[0, h_{i}\right]$, it has a finite number of roots, none of which are on the imaginary axis. We conclude that for any fixed $\tau_{i} \in\left[0, h_{i}\right]$,

$$
\inf _{\omega \geq 0}\left\|\sum_{i=0}^{n} q_{i}(\omega \imath) R_{m}\left(\alpha_{m} \tau_{i} \omega \imath\right)\right\|>0
$$

Therefore, since the $\left[0, h_{i}\right]$ are compact sets,

$$
r_{m}:=\inf \left\{\left\|\sum_{i=0}^{n} q_{i}(\omega \imath) R_{m}\left(\alpha_{m} \tau_{i} \omega \imath\right)\right\|: \omega \geq 0, \tau_{i} \in\left[0, h_{i}\right]\right\}>0 .
$$

Therefore, by Lemma 15

$$
r_{e}:=\inf \left\{\left\|\sum_{i=0}^{n} q_{i}(\omega \imath) e^{-\tau_{i} \omega \imath}\right\|: \omega \geq 0, \tau_{i} \in\left[0, h_{i}\right]\right\} \geq r_{m}>0 .
$$

This proves that

$$
\left\|\operatorname{det} G_{\tau}^{-1}\right\|_{\infty}=\sup _{\omega \geq 0}\left\|\operatorname{det} G_{\tau}(\imath \omega)\right\|^{-1} \leq \frac{1}{r_{m}}
$$

and so $\operatorname{det} G_{\tau}(s)^{-1} \in H_{\infty}$ for any $\tau_{i} \in\left[0, h_{i}\right]$. 


\subsection{Delay-Independent Stability}

To check stability independent of delay, the conditions of Theorem 4 may be modified slightly by considering the semialgebraic set

$$
\left\{\omega, \tau_{i} \in \mathbb{R}: \omega \geq 0, \tau_{i} \geq 0, g_{r}\left(\omega, \tau_{i}\right)=0, g_{i}\left(\omega, \tau_{i}\right)=0\right\}
$$

In this section, we propose an alternative approach which eliminates the need for potentially high-order rational approximations. We begin by noting the following proposition which gives conditions that, when combined with the results of Datko, can be used to prove that no poles enter the right half-plane for any value of delay.

Proposition 1. The following conditions are equivalent

- $\quad$ The set $\left\{\omega \in \mathbb{R}, \tau \in \mathbb{R}^{k}: \omega \neq 0, \tau_{k} \geq 0\right.$ for $\left.k=1, \ldots, n, \sum_{k=0}^{n} q_{k}(\imath \omega) e^{-\imath \omega \tau_{k}}=0\right\}$ is empty.

- $\quad$ The set $\left\{\omega \in \mathbb{R}, \tau \in \mathbb{C}^{k}: \omega \neq 0,\left|z_{k}\right|=1, \sum_{k=0}^{n} q_{k}(s) z_{k}=0\right\}$ is empty.

Proof. We prove 2) $\Longrightarrow 1$ ) by contrapositive. Suppose 1) is false for some $\omega_{0}, \tau_{k}$. Then let $z_{k}=e^{-\tau_{k} \omega_{0} \imath} \omega=\omega_{0}$, which is feasible for 2). Therefore, by contrapositive 2) $\Longrightarrow 1$ ).

For 1) $\Longrightarrow 2$ ), suppose 2) is false. Then there exists $\omega_{0} \neq 0$ and $z \in \mathbb{C}^{k}$ with $\left|z_{k}\right|=1$ such that $\sum_{k=0}^{n} q_{k}\left(\imath \omega_{0}\right) z_{k}=0$. We can write $z_{k}=e^{-\imath a_{k}}$ with sign $a_{k}=\operatorname{sign} \omega_{0}$. Let $\tau_{k}=\frac{a_{k}}{\omega_{0}}$ and $\omega=\omega_{0}$. Then we get

$$
\sum_{k=0}^{n} q_{k}(\imath \omega) e^{-\imath \omega \tau_{k}}=\sum_{k=0}^{n} q_{k}\left(\imath \omega_{0}\right) e^{-\imath \omega_{0} \tau_{k}}=\sum_{k=0}^{n} q_{k}\left(\imath \omega_{0}\right) e^{-\imath a_{k}}=\sum_{k=0}^{n} q_{k}\left(\imath \omega_{0}\right) z_{k}=0 .
$$

This contradicts 1) and completes the proof.

Proposition 1 is incomplete in that it does not prove $H_{\infty}$ or exponential stability. This is because neutral-type delay systems can have infinite roots which may approach the imaginary axis, creating an unbounded high frequency response. This problem was avoided in the previous section by using rational approximations. To deal with this issue, we consider the case of commensurate delays. This is made explicit by the following Lemma.

Lemma 1. The following conditions are equivalent

1. The set $\left\{s \in \imath \mathbb{R}^{+}, \tau_{k} \in \mathbb{R}^{+}: \sum_{k=0}^{n} q_{k}(s) e^{-s k \tau}=0\right\}$ is empty.

2. The following semialgebraic set is empty

$$
\begin{aligned}
\left\{\omega, z_{i}, z_{r} \in \mathbb{R}: 1-z_{i}^{2}+z_{r}^{2}=0,\right. & \operatorname{Re}\left(\sum_{k=0}^{n} q_{k}(\imath \omega)\left(z_{r}+\imath z_{i}\right)^{k}\right)=0, \\
& \left.\operatorname{Im}\left(\sum_{k=0}^{n} q_{k}(\imath \omega)\left(z_{r}+\imath z_{i}\right)^{k}\right)=0\right\}
\end{aligned}
$$


3. There exist polynomials $t_{1}, t_{2}, t_{3} \in \mathbb{R}\left[\omega, z_{r}, z_{i}\right]$ and $s \in \Sigma_{s}$ such that

$$
\begin{aligned}
-1= & \left(z_{r}^{2}+z_{i}^{2}-1\right) t_{1}-\operatorname{Re}\left(\sum_{k=0}^{n} q_{k}(\imath \omega)\left(z_{r}+\imath z_{i}\right)^{k}\right) t_{2} \\
& +\operatorname{Im}\left(\sum_{k=0}^{n} q_{k}(\imath \omega)\left(z_{r}+\imath z_{i}\right)^{k}\right) t_{3}+s .
\end{aligned}
$$

The proof is an application of Theorem 1 This leads to a simple condition for stability of neutral systems with commensurate delays.

Theorem 5. Suppose $G_{\tau}$, as given by Equation (2), satisfies the conditions of Theorem 2 and $\operatorname{det} G_{\tau}(s):=\sum_{k=0}^{n} q_{i}(s) e^{-k \tau s}$. Suppose that

$$
\left\{s \in \mathcal{C}: \sum_{i=0}^{n} q_{i}(s)=0, \operatorname{Re} s \geq 0\right\}=\emptyset
$$

and that there exist polynomials $t_{1}, t_{2}, t_{3} \in \mathbb{R}\left[\omega, z_{r}, z_{i}\right]$ and $s \in \Sigma_{s}$ such that

$$
\begin{aligned}
-1= & \left(z_{r}^{2}+z_{i}^{2}-1\right) t_{1}-\operatorname{Re}\left(\sum_{k=0}^{n} q_{k}(\imath \omega)\left(z_{r}+\imath z_{i}\right)^{k}\right) t_{2} \\
& +\operatorname{Im}\left(\sum_{k=0}^{n} q_{k}(\imath \omega)\left(z_{r}+\imath z_{i}\right)^{k}\right) t_{3}+s .
\end{aligned}
$$

Then the system defined by Equation 1 is both $H_{\infty}$ and exponentially stable for all $\tau \geq 0$

Proof. The proof is similar to that of Theorem 4 By combining Theorem 2 Lemma 1 and the assumption, $G_{\tau}$ has no roots on the imaginary axis for any positive value of $\tau$. Moreover, for the case of commensurate delays, it can be shown that the conditions of Theorem 2 imply that a lack of roots in the closed right half-plane implies both exponential and $H_{\infty}$ stability.

\subsection{Verifying the Datko Conditions}

In this section, we briefly consider the problem of verifying the conditions associated with Theorem 2 In particular, we would like to show that

$$
\operatorname{det}\left[I-\sum_{i=1}^{m} B_{i} e^{-s \gamma_{i}}\right]=0
$$

has all roots lying in some left half-plane $\operatorname{Re} s \in\left(-\infty,-\beta_{0}\right], \beta_{0}>0$.

A simple sufficient condition, also proposed in [3], is given by the following.

Proposition 16. Suppose $B_{i}=0$ for $i=1, \ldots m-1$ and $\operatorname{det}\left(\lambda I-B_{m}\right)$ has all roots in the $\operatorname{disc}|\lambda|<1$. Then the conditions of Theorem 2 are satisfied.

More generally, if the delays are commensurate, then the condition is equivalent to exponential stability of an expanded discrete-time linear system of the general form

$$
\left[\begin{array}{c}
x_{1}(k+1) \\
\vdots \\
x_{n}(k+1)
\end{array}\right]=\left[\begin{array}{ccc}
B_{1} & \cdots & B_{n} \\
I & \\
& I
\end{array}\right]\left[\begin{array}{c}
x_{1}(k) \\
\vdots \\
x_{n}(k)
\end{array}\right] .
$$

If the delays are non-commensurate, then an SOS condition can also be given. 
Lemma 17. Let $\epsilon>0$. If

$$
\left\{z_{i} \in \mathbb{C}: \operatorname{det}\left(I+\sum_{i=1}^{m} B_{i} z_{i}\right)=0,\left|z_{i}\right| \leq e^{\epsilon T_{i}}\right\}=\emptyset
$$

then

$$
\operatorname{det}\left[I+\sum_{i=1}^{m} B_{i} e^{-s \gamma_{i}}\right]=0
$$

has all roots in the left half-plane $\{\operatorname{Re} s \leq-\epsilon\}$ for all $\gamma_{i} \leq T_{i}$.

Proof. Proof by contradiction. Suppose that there exist $\left(\gamma_{1}, \cdots, \gamma_{n}\right) \in \mathbb{R}^{n}$ with $\gamma_{i} \leq T_{i}$ for $i=1, \cdots, m$ and $s_{0} \in\{\operatorname{Re} s \geq-\epsilon\}$ such that

$$
\operatorname{det}\left(I+\sum_{i=1}^{m} B_{i} e^{-\gamma_{i} s_{0}}\right)=0 \text {. }
$$

Let $s=s_{0}$ and $z_{i}=e^{-\gamma_{i} s_{0}}$. Then $s_{0} \in\{\operatorname{Re} s \geq-\epsilon\}$ and $\left|z_{i}\right| \leq e^{\epsilon T_{i}}$ with

$$
\operatorname{det}\left(I+\sum_{i=1}^{n} B_{i} z_{i}\right)=0
$$

which contradicts the statement of the lemma.

The conditions of Lemma 17 can be verified using SOS by application of Theorem 1

\section{Numerical Example}

Table 1. Stability Regions

$$
m=3 m=4 m=5 \text { actual value }
$$

\begin{tabular}{lllll} 
maximum $\tau$ & 1.805 & 2.157 & 2.217 & 2.2255 \\
required degree & 14 & 18 & 22 & \\
\hline
\end{tabular}

Example 1: In this example, we consider a somewhat arbitrarily chosen example to illustrate the delay-dependent stability condition associated with Theoren 4 . We use the example chosen by [4], [6], and [5] among many others.

$$
\dot{x}(t)-B \dot{x}(t-\tau)=A_{0} x(t)+A_{1} x(t-\tau)
$$

where

$$
\begin{aligned}
& A_{0}=\left[\begin{array}{cc}
-0.9 & 0.2 \\
0.1 & -0.9
\end{array}\right], \quad A_{1}=\left[\begin{array}{ll}
-1.1-0.2 \\
-0.1-1.1
\end{array}\right], \\
& B=\left[\begin{array}{cc}
-0.2 & 0 \\
0.2 & -0.1
\end{array}\right] .
\end{aligned}
$$


To test stability, we first verify the Datko conditions, which hold by Proposition 16 We now replace the characteristic equation

$$
g(s)=\operatorname{det}\left(s I-A_{0}-A_{1} e^{-\tau s}-B s e^{-\tau s}\right)
$$

with the family of rational approximations $R_{m}$ to get a new characteristic polynomial family

$$
\begin{aligned}
H_{m}(s, \tau)= & \operatorname{det}\left(s P_{m}\left(-\alpha_{m} \tau s\right) I-A_{0} P_{m}\left(-\alpha_{m} \tau s\right)\right. \\
& \left.-A_{1} P_{m}\left(\alpha_{m} \tau s\right)-B s P_{m}\left(\alpha_{m} \tau s\right)\right)
\end{aligned}
$$

We then create the real polynomial functions

$$
g_{i}(\omega, \tau):=\operatorname{Re} H_{m}(\imath \omega, \tau)
$$

and

$$
g_{r}(\omega, \tau):=\operatorname{Re} H_{m}(\imath \omega, \tau)
$$

This can be done automatically in Matlab using the function cpoly2rpoly contained in the software package available online at [8].

We now use SOSTools [7] to find polynomials $t_{1}, t_{2} \in \mathbb{R}[\omega, \tau]$ and SOS polynomials $s_{i} \in \Sigma_{s}[\omega, \tau]$ for $i=0, \ldots, 3$ such that

$$
-1=s_{0}+t_{1} g_{r}+t_{2} g_{i}+\omega s_{1}+\tau(h-\tau) s_{2}+\omega \tau(h-\tau) s_{3}
$$

By using this method, we are able to prove stability of the system for the values of delay listed in Table 1 This table also lists the degree of the refutation necessary. The accuracy is roughly comparable to what is currently available using existing time-domain methods. Note that the accuracy is restricted only by the value of $\alpha_{m}$.

\section{Conclusion}

We have a proposed a method to check delay-dependant stability of neutral-type delay systems which involves the Padé approximate of $e^{-s}$ and uses sum-of-squares methods to prove the infeasibility of certain semi-algebraic sets. The method is applied to a standard example from the literature.

\section{References}

1. Bliman, P.A., Iwasaki, T.: LMI characterisation of robust stability for time-delay systems: singular perturbation approach. In: Proc. IEEE Conference on Decision and Control, San Diego, USA (2006)

2. Bonnet, C., Peet, M.M.: Using the positivstellensatz for stability analysis of neutral delay systems in the Frequency Domain. In: Proc. 7th IFAC Workshop on Time-Delay Systems, Nantes, France (2007)

3. Datko, R.: A procedure for determination of the exponential stability of certain differential-difference equations. Quarterly of Applied Mathematics 36, 279-292 (1978)

4. Fridman, E.: New Lyapunov-Krasovskii functionals for stability of linear retarded and neutral-type systems. Automatica 43, 309-319 (2001) 
5. Han, Q.L.: On stability of linear neutral systems with mixed delays: a discretized Lyapunov functional approach. Automatica 41(7), 1209-1218 (2005)

6. He, Y., Wu, M., She, J.H., Liu, G.P.: Delay-dependent robust stability criteria for uncertain neutral systems with mixed delays. Systems \& Controls Letters 51(1), 57-65 (2004)

7. Parrilo, P.A.: Web Site for SOSTOOLS (2004), http: //www.cds.caltech.edu/sostools

8. Peet, M.: Web Site for Matthew M. Peet.

Url:http: http: / /www-rocq.inria.fr/ peet

9. Stengle, G.: A nullstellensatz and a positivstellensatz in semialgebraic geometry. Mathematische Annalen 207, 87-97 (1973)

10. Zhang, J., Knospe, C., Tsiotras, P.: Stability of linear time-delay systems: a delaydependant criterion with a tight conservatism bound. In: Proc. American Control Conference, Anchorage, USA (2002) 